\title{
Hispanic Patients with Primary Biliary Cholangitis Have Decreased Access to Care Compared to Non-Hispanics
}

\author{
Atoosa Rabiee*1, Nathalie A Pena Polanco ${ }^{2}$, Aymara Fernandez De La Vara ${ }^{3}$ and Cynthia Levy ${ }^{2}$ \\ ${ }^{1}$ Department of Gastroenterology and Hepatology, VA Medical Center, Washington DC, USA; ${ }^{2}$ Divison of Digestive Health and \\ Liver Diseases Department of Medicine University of Miami Miller School of Medicine USA; ${ }^{3}$ Department of Medicine, Jackson \\ Memorial Hospital, University of Miami, Miami, FL, USA
}

\begin{abstract}
Background and Aims: Hispanic patients with primary biliary cholangitis (PBC) have reduced rates of biochemical response to ursodeoxycholic acid (UDCA) and increased risk of disease progression compared to non-Hispanic patients. In this study, we sought to identify differences in demographics, comorbidities, environmental risk factors and socioeconomic status between Hispanic and non-Hispanic patients with PBC. Methods: In a case control study, we analyzed data from Hispanic ( $n=37$ females and 1 male) and non-Hispanic ( $n=54$ females and 4 males) patients with PBC seen at the University of Miami/Jackson Memorial Hospital from January 1998 through January 2013. Data were obtained by filling out a questionnaire either via phone call, mail, or e-mail. Odds ratios were calculated to measure the association between exposure and outcomes.Results: Baseline demographics, environmental risk factors and comorbidities were similar between Hispanic and non-Hispanic patients with PBC. Hispanic patients were less likely to be married and fewer Hispanics had education beyond high school level compared to non-Hispanics. Sixty four percent of Hispanic patients had a household income of less than $\$ 50000$, compared to $19.5 \%$ of nonHispanics. Fewer Hispanic patients with PBC had health insurance coverage compared to non-Hispanics (86.5\% vs. 98.1\%; odds ratio: $0.1,95 \%$ confidence interval: $0-0.9$ ). Conclusions: Differences in disease severity and response to therapy observed in prior studies could not be explained by environmental exposures. In addition to genetic variation, socioeconomic discrepancies (access to care) may further explain these differences.

Citation of this article: Rabiee A, Pena Polanco NA, De La Vara $A F$, Levy $C$. Hispanic patients with primary biliary cholangitis have decreased access to care compared to non-Hispanics. J Clin Transl Hepatol 2020;8(4):391-396. doi: 10.14218/JCTH.2020.00006.
\end{abstract}

Keywords: Primary biliary cholangitis; Ethnicity; Health disparity.

Abbreviations: AASLD, American Association for the study of Liver Diseases; ALP, alkaline phosphatase; CI, confidence interval; NAFLD, nonalcoholic fatty liver disease; OR, odds ratio; PBC, primary biliary cholangitis; UDCA, ursodeoxycholic acid.

Received: 28 January 2020; Revised: 21 July 2020; Accepted: 18 September 2020

*Correspondence to: Atoosa Rabiee, Department of Gastroenterology and Hepatology, VA Medical Center, 50 Irving St NW, Washington DC 20422, USA. Tel: +1202-745-8456, E-mail: rabiee.atoosa@gmail.com

\section{Introduction}

Primary biliary cholangitis (PBC) is a chronic immune-mediated liver disease characterized by inflammation and progressive loss of small intrahepatic bile ducts, eventually leading to biliary cirrhosis. PBC is a rare disease, affecting mainly women in their middle age, and the exact pathogenesis remains incompletely understood. Little information is available regarding the effect of race or ethnicity on susceptibility or progression of PBC.

A multicenter study by Peters et al. ${ }^{1}$ described the differences in severity and disease progression by race and ethnicity in patients with PBC. The study concluded that progression to advanced liver disease, ascites, hepatic encephalopathy, and variceal bleeding was more frequent in the non-Caucasian group. Non-Caucasians also had lower levels of physical activity and more severe pruritus. Notably, among the 535 patients enrolled in the study, $86.4 \%$ were Caucasians. Non-Caucasians included 42 (7.9\%) Hispanics, 21 (3.9\%) African Americans, and $10(1.8 \%)$ other. We have previously shown in a cross-sectional study that Hispanic patients with PBC have reduced response rates to UDCA. ${ }^{2}$ In that study, Hispanic patients were more likely to have complications of advanced liver disease, such as portal hypertension, as compared to non-Hispanics. The reasons for such disparities were not addressed.

In the present study, we aimed to evaluate the demographics, comorbidities, environmental risk factors and socioeconomic status of Hispanic patients with PBC compared to non-Hispanic ones.

\section{Methods}

We identified all patients with diagnosis of PBC seen at the University of Miami/Jackson Memorial Hospital (Miami, FL, USA) between January 1998 and January 2013, through ICD9 codes. Diagnosis of PBC was confirmed by chart review. As recommended by the American Association for the study of Liver Diseases (AASLD), diagnosis of PBC was confirmed when patients met two out of the following three criteria: 1 ) chronic cholestasis, evidenced by persistent increase in serum alkaline phosphatase (ALP); 2) positive serology for anti-mitochondrial antibodies; and 3 ) histologic confirmation of PBC, with the presence of nonsuppurative destructive cholangitis involving interlobular and septal bile ducts.

We developed a questionnaire-based survey, available in English and Spanish, to evaluate patients' demographics, comorbid conditions, environmental risk factors and 
socioeconomic status, and contacted patients by mail, e-mail, or phone call. A pre-designed consent form was read over the phone to patients, and a verbal consent was obtained prior to collection of information. If the questionnaire was sent by email or mail, this form was included for patients to review, and return of questionnaire implied that consent was provided. We collected data on patients' demographics, clinical presentation, comorbidities, risk factors for PBC, and socio-economic variables.

Once patients were identified and diagnosis confirmed by chart review, they were contacted via telephone call, e-mail communication, or postal mail. Multiple attempts were made to reach each patient. Of 265 subjects with confirmed diagnosis, 10 were deceased, 5 did not want to participate, and 26 had incorrect contact information in the medical records and could not be reached. Thus, the study population consisted of 224 patients with confirmed PBC. The study protocol and questionnaires were approved by the University of Miami Institutional Review Board.

\section{Statistical analysis}

Descriptive statistics were used to characterize the study population. Results were provided as $n(\%)$ or median (interquartile range). Odds ratios (ORs) and their $95 \%$ confidence intervals (Cis) were calculated to measure the association between exposure and outcome.

Evidence in favor of an association was determined by the exclusion of 1 from this interval. Male patients were excluded from the data analysis, since there were so few of them $(n=5)$, to preserve homogeneity of the sample and generalizability of the result to the majority of PBC patients who are female. All analyses were performed using R (version 3.2.2) and the 'epitools' package. ${ }^{3}$

\section{Results}

\section{Demographics}

Two hundred and twenty-four patients were given the questionnaire and ninety-six (43\%) completed it (Fig. 1). Of the responders, $39.6 \%$ were Hispanic (38 in total; 37 females and 1 male) and $60.4 \%$ were non-Hispanic patients (58 in total; 54 females and 4 males); forty-nine (91\%) in the non-Hispanic group were White, 3.7\% Black, 1.9\% American Indian, and $3.7 \%$ other. Hispanic patients were all White. Median age of Hispanic patients was 51 years-old (age range: 30-75). Non-Hispanics had a median age of 53.5 (age range: 28-74). Among Hispanics, 58.3\% were married, as compared to $75.4 \%$ of non-Hispanic patients. one patient had prior hepatitis $\mathrm{B}$ exposure and one had prior HCV infection; both were non-Hispanics. Twenty-six patients had radiographic evidence of cirrhosis; among them, $54 \%$ of these were Hispanic $(n=14)$. Table 1 summarizes the demographics of our study population.

Among Hispanic patients, $45.9 \%$ were from the Caribbean, $32.4 \%$ from South America, and $10.8 \%$ from Central America; only four were born in the United States. The vast majority $(83.8 \%)$ have lived in the USA for more than 15 years. Hispanic patients were diagnosed in a more recent era than the non-Hispanics. Ninety-two percent of Hispanic patients were diagnosed after year 2000, as compared to $42.6 \%$ of non-Hispanics.

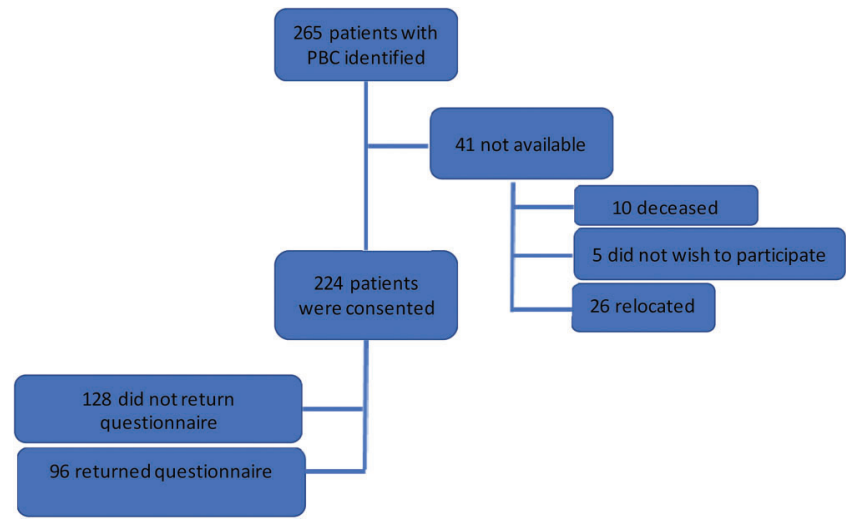

Fig. 1. Flow chart showing study enrollment.

Table 1. Baseline characteristics of Hispanic and non-Hispanic patients with PBC

\begin{tabular}{|c|c|c|}
\hline Baseline characteristics & $\begin{array}{l}\text { Hispanics, } \\
n=37\end{array}$ & $\begin{array}{l}\text { Non- } \\
\text { Hispanics, } \\
n=54\end{array}$ \\
\hline Age, median (range) & $51(30-75)$ & $\begin{array}{l}53.5(28- \\
74)\end{array}$ \\
\hline \multicolumn{3}{|l|}{ Race, $n(\%)$} \\
\hline White & $38(100 \%)$ & $49(90.7 \%)$ \\
\hline Black & & $2(3.7 \%)$ \\
\hline Asian & & $1(1.9 \%)$ \\
\hline Other & & $2(3.7 \%)$ \\
\hline \multicolumn{3}{|l|}{ Marital status, $n(\%)$} \\
\hline Single & $10(27.8 \%)$ & $6(11.3 \%)$ \\
\hline Married & $21(58.3 \%)$ & $40(75.4 \%)$ \\
\hline Other & $5(13.9 \%)$ & $7(13.2 \%)$ \\
\hline \multicolumn{3}{|l|}{$\begin{array}{l}\text { Years lived in the USA, } n \\
(\%)\end{array}$} \\
\hline Less than 15 years & $6(16.2 \%)$ & 0 \\
\hline 15 years or more & $31(83.8 \%)$ & $53(100 \%)$ \\
\hline $\begin{array}{l}\text { Year of diagnosis, median } \\
\text { (interquartile range) }\end{array}$ & $\begin{array}{l}2007 \\
(2005- \\
2008)\end{array}$ & $\begin{array}{l}2002 \\
(1997- \\
2002)\end{array}$ \\
\hline \multicolumn{3}{|l|}{$\begin{array}{l}\text { Place of living at the time of } \\
\text { diagnosis, } n(\%)\end{array}$} \\
\hline USA & $36(97.3 \%)$ & $86(100 \%)$ \\
\hline Outside of USA & $1(2.7 \%)$ & 0 \\
\hline $\begin{array}{l}\text { Years lived in the location } \\
\text { prior to diagnosis, median } \\
\text { (range) }\end{array}$ & $18.6(2-44)$ & $22.7(1-55)$ \\
\hline
\end{tabular}

Hispanic patients were diagnosed with PBC mainly when living in the USA. Only $2.7 \%$ of Hispanic patients had been diagnosed outside of the USA. Average years lived in the location where patients were diagnosed with PBC was 18.7 years (range: 2-64) and 22.7 years (range: 1-62) for Hispanic and non-Hispanic patients, respectively. 
Rabiee A. et al: Health disparity in Hispanic PBC patients

\section{Comorbidities and potential risk factors}

There were no differences between groups with respect to hair dye and nail polish use, alcohol and tobacco use, age of menarche or menopause, number of miscarriages or pregnancies, or itching during pregnancy. Although non-Hispanics were more likely to use birth control pills or hormone replacement therapy, this difference did not reach statistical significance. There was no statistically different difference in history of urinary tract infections, vaginal infections, sexually transmitted diseases, concomitant medical conditions, or surgical procedures (Fig. 2). Symptoms at the time of presentation, diagnosis of PBC or autoimmune disease in a first degree relative were also not statistically significant between the two groups. Table 2 details the frequency of symptoms, comorbidities and risk factors in the two Ethnic groups.

\section{Socioeconomic factors}

In our study, $27 \%$ of Hispanic patients had a high school education or less as the highest level of education, as compared to only $17 \%$ of non-Hispanics. There was no statistically significant difference in the employment status between the two groups ( $83.3 \%$ of Hispanics were employed, as compared to $92.3 \%$ of non-Hispanics). Among Hispanics, $62.8 \%$ had a household income of less than $\$ 50,000$, as compared to only $19.5 \%$ of non-Hispanics (OR: $6.7,95 \% \mathrm{CI}$ : 2.5-19.2). Most patients in both Hispanic and non-Hispanic groups were not the sole provider of income in the family. The median number of family members supported by the family income was the same in both the Hispanic and non-Hispanic groups. Only $1.9 \%$ of non-Hispanics did not have any health care insurance, as compared to $13.5 \%$ of Hispanics (OR: 0.1 , 95\% CI: 0-0.9) (Table 3).

\section{Treatment with UDCA}

Median dose of UDCA was the same for both Hispanic and non-Hispanic groups $(1000 \mathrm{mg})$. Hispanic patients were numerically more likely to miss their medications, as compared to non-Hispanics, but this did not reach statistical significance (35.5\% vs. $22.6 \%$ ). The proportion of patients going without medications to afford food, housing and bills was similar in both groups $(82.9 \%$ in Hispanics vs. $87 \%$ in non-Hispanics).

\section{Discussion}

In the current study, we investigated differences in demographics, comorbidities, environmental risk factors and socioeconomic status between Hispanic and non-Hispanic patients with PBC. We could not demonstrate any significant difference in comorbidities or environmental risk factors between the two groups. However, we found important socio-economic disparities, including lower level of education, lower income, and decreased availability of health care insurance among Hispanic patients. Furthermore, Hispanic patients were less likely to be married and frequently had a "recent" diagnosis of PBC compared to the Non-Hispanic patients. All of these characteristics could have a major role in disease progression and their medical management.

Carrion et al., ${ }^{4}$ studied chronic liver disease with different etiologies in Hispanic patients. The investigators found higher incidence and more aggressive course as well as worse outcomes despite treatment for many of these diseases. Specifically, Hispanic patients with nonalcoholic fatty liver disease (commonly known as NAFLD) had more advanced fibrosis and Hispanic patients with chronic hepatitis $C$ infection had faster progression to cirrhosis. Furthermore, incidence and mortality from hepatocellular carcinoma were higher in Hispanics compared to non-Hispanic whites.

Peters et al. ${ }^{1}$ studied patients with PBC from 11 of the states in the USA, including 501 females and 34 males, mean age of 52, from an ethnically diverse background. In that study, most patients were Caucasian, although their cohort also included 42 Hispanics. They showed more severe disease in Hispanics and African Americans, with greater limitation on activity level, worse pruritus, and

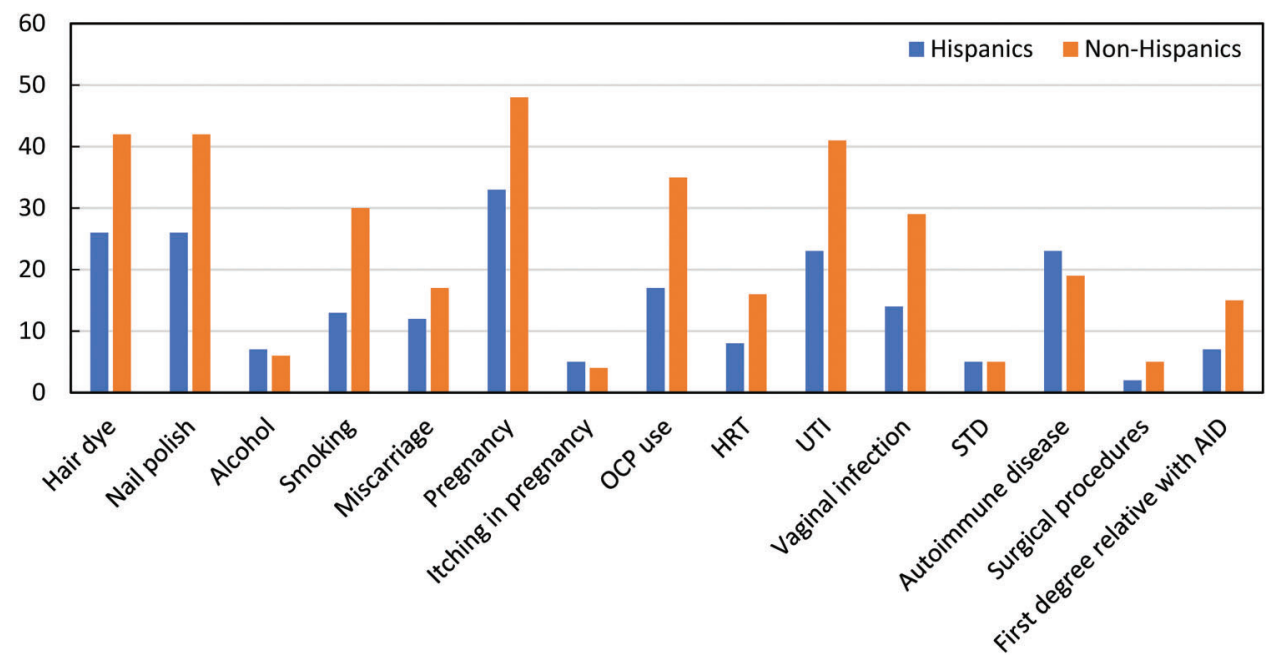

Fig. 2. Comorbidities and risk factors in Hispanic patients with PBC compared with non-Hispanics.

Abbreviation: AID, autoimmune disease; HRT, hormone replacement therapy; UTI, urinary tract infection; STD, sexually transmitted disease; PBC, primary biliary cholangitis. 
Rabiee A. et al: Health disparity in Hispanic PBC patients

Table 2. Comorbidities and risk factors in Hispanic and non-Hispanic patients with PBC

\begin{tabular}{|c|c|c|c|}
\hline Comorbidities and risk factors & Hispanics & Non-Hispanics & OR $(95 \% \mathrm{CI})$ \\
\hline \multicolumn{4}{|l|}{ Hair dye, $n(\%)$} \\
\hline Never, rarely & $11(30 \%)$ & $12(23 \%)$ & $1.48(0.56-3.89)$ \\
\hline Sometimes, often, always & $26(70 \%)$ & $42(77 \%)$ & \\
\hline \multicolumn{4}{|l|}{ Nail polish, $n(\%)$} \\
\hline Never, rarely & $11(29.7 \%)$ & $12(22.2 \%)$ & $1.47(0.56-3.89)$ \\
\hline Sometimes, often, always & $26(70.3 \%)$ & $42(77.8 \%)$ & \\
\hline \multicolumn{4}{|l|}{ Tanning salon use, $n(\%)$} \\
\hline Never, rarely & $37(100 \%)$ & $54(100 \%)$ & \\
\hline Sometimes, often, always & 0 & 0 & \\
\hline \multicolumn{4}{|l|}{ Alcohol use, $n(\%)$} \\
\hline Never, rarely & $30(81.1 \%)$ & $48(88.9 \%)$ & $0.54(0.16-1.82)$ \\
\hline Sometimes, often, always & $7(18.9 \%)$ & $6(11.1 \%)$ & \\
\hline \multicolumn{4}{|l|}{ Smoking, $n(\%)$} \\
\hline Never, rarely & $22(62.9 \%)$ & $23(43.4 \%)$ & $2.18(0.91-5.37)$ \\
\hline Sometimes, often, always & $13(37.1 \%)$ & $30(56.6 \%)$ & \\
\hline Age of menarche, median (range) & $12(9-20)$ & $12(10-15)$ & \\
\hline Age of menopause, median (range) & $48(37-60)$ & $50(35-60)$ & \\
\hline Number of living children, median (range) & $2(0-5)$ & $2(0-4)$ & \\
\hline History of miscarriage, $n(\%)$ & $12(33.3 \%)$ & $17(32.7 \%)$ & $1.03(0.41-2.56)$ \\
\hline History of pregnancy, $n(\%)$ & $33(91.7 \%)$ & $48(92.3 \%)$ & $0.91(0.18-5.20)$ \\
\hline History of itching during pregnancy, $n(\%)$ & $5(16.1 \%)$ & $4(9.5 \%)$ & $1.79(0.42-8.24)$ \\
\hline History of birth control use, $n(\%)$ & $17(45.9 \%)$ & $35(66 \%)$ & $0.44(0.18-1.05)$ \\
\hline $\mathrm{HRT}, n(\%)$ & $8(21.6 \%)$ & $16(30.2 \%)$ & $0.64(0.23-1.70)$ \\
\hline UTI, $n(\%)$ & $23(62.2 \%)$ & $41(75.9 \%)$ & $0.52(0.21-1.32)$ \\
\hline Vaginal infection, $n(\%)$ & $14(38.9 \%)$ & $29(55.8 \%)$ & $0.51(0.21-1.21)$ \\
\hline STD, $n(\%)$ & $5(13.9 \%)$ & $5(9.6 \%)$ & $1.51(0.38-6.05)$ \\
\hline Comorbidity, $n(\%)^{*}$ & $8(21.6 \%)$ & $10(18.5 \%)$ & $1.21(0.41-3.49)$ \\
\hline Surgery, $n(\%)$ & $2(5.4 \%)$ & $5(9.3 \%)$ & $0.59(0.07-3.03)$ \\
\hline Itching at diagnosis, $n(\%)$ & $15(40.5 \%)$ & $26(50.0 \%)$ & $0.69(0.29-1.61)$ \\
\hline First degree relative with $\mathrm{PBC}, n(\%)$ & $7(18.9 \%)$ & $15(32.6 \%)$ & $0.49(0.16-1.35)$ \\
\hline
\end{tabular}

Abbreviations: HRT, hormone replacement therapy; UTI, urinary tract infection; STD, sexually transmitted disease.

*Comorbidity includes Sjogren's syndrome, hypothyroidism, scleroderma, CREST, Raynaud's, rheumatoid arthritis, systemic lupus erythmatosos, polymyositis, diabetes, hypertension, coronary artery disease, hypercholesterolemia, history of cancer, asthma, herpes zoster, celiac disease.

severe disease as evidenced by laboratory data and exam findings (including splenomegaly, telangiectasia, peripheral edema, icterus, ascites and clubbing). Along these lines, Hispanics and African Americans were more likely to be excluded from treatment trials due to more severe liver disease. The investigators postulated that this increased disease severity noted among Hispanics and African Americans could have been due to delayed referral to tertiary care center, although this could not be evaluated. Another hypothesis was that Hispanics and African Americans had earlier onset PBC and were misdiagnosed. It was unclear from the study if these patients had more limited access to care, faster disease progression, initial misdiagnosis, or different comorbidities affecting their progress.

Although we have previously shown that Hispanic patients with PBC are more likely to have complications of advanced liver disease ${ }^{2}$ the potential factors contributing to this observation were not studied. Thus, we were interested in understanding how one's ethnicity and social environment could influence the clinical presentation and progression of PBC. Potential environmental risk factors affecting development of PBC have been extensively evaluated and include smoking, ${ }^{5-9}$ tonsillectomy, ${ }^{5}$ vaginal or urinary tract infections (especially if multiple), ${ }^{5-9}$ having a first degree relative with $\mathrm{PBC}^{6,7,9}$ use of hormone replacement therapy, ${ }^{7}$ history of psoriasis and other autoimmune diseases, ${ }^{6,8,9}$ and shingles. ${ }^{8}$ Prince et al. ${ }^{8}$ found no association with obstetric risk factors. Interestingly, in one study, the longer use of oral contraceptive pills was actually protective against development of $\mathrm{PBC}^{6}$ In addition to being a risk factor for the development of PBC, smoking is also an independent risk factor for development of fibrosis in PBC patients. ${ }^{10}$ 
Rabiee A. et al: Health disparity in Hispanic PBC patients

Table 3. Socioeconomic characteristics of Hispanic and non-Hispanic patients with $\mathrm{PBC}$

\begin{tabular}{|c|c|c|c|}
\hline $\begin{array}{l}\text { Socioeconomic } \\
\text { variables }\end{array}$ & Hispanics & $\begin{array}{l}\text { Non- } \\
\text { Hispanics }\end{array}$ & $\begin{array}{l}\text { OR }(95 \% \\
\text { CI })\end{array}$ \\
\hline $\begin{array}{l}\text { High School } \\
\text { education and } \\
\text { below } \\
\text { College } \\
\text { education and } \\
\text { above }\end{array}$ & $\begin{array}{l}27 \% \\
73 \%\end{array}$ & $\begin{array}{l}17 \% \\
83 \%\end{array}$ & $\begin{array}{l}1.7 \\
(0.6-5.1)\end{array}$ \\
\hline $\begin{array}{l}\text { Employed } \\
\text { Unemployed }\end{array}$ & $\begin{array}{l}83.3 \% \\
16.7 \%\end{array}$ & $\begin{array}{l}92.3 \% \\
7.7 \%\end{array}$ & $\begin{array}{l}2.3 \\
(0.6-10.2)\end{array}$ \\
\hline $\begin{array}{l}\text { Household } \\
\text { income less than } \\
50,000\end{array}$ & $62.9 \%$ & $19.6 \%$ & $\begin{array}{l}6.7 \\
(2.5-19.2)^{*}\end{array}$ \\
\hline $\begin{array}{l}\text { Health care } \\
\text { insurance }\end{array}$ & $86.5 \%$ & $98.1 \%$ & $\begin{array}{l}0.1 \\
(0-0.9) *\end{array}$ \\
\hline $\begin{array}{l}\text { Missing } \\
\text { medications }\end{array}$ & $35.5 \%$ & $22.6 \%$ & $\begin{array}{l}0.5 \\
(0.2-1.4)\end{array}$ \\
\hline $\begin{array}{l}\text { Missing } \\
\text { medications to } \\
\text { afford essentials }\end{array}$ & $17.1 \%$ & $13 \%$ & $\begin{array}{l}1.3 \\
(0.4-4.6)\end{array}$ \\
\hline
\end{tabular}

Data from recent studies also indicates that Hispanic patients with PBC are less likely to undergo liver transplantation than Caucasian patients. Using the United Network for Organ Sharing registry, Cholankeril et al. ${ }^{11}$ performed a retrospective review of the PBC liver transplant waitlist registrant's cohort, from 2000 to 2014. During this period, of a total of 156,624 adult patients listed for liver transplant, only $3.5 \%$ were waitlisted for a primary diagnosis of PBC. Overall, their mean age was 55.6 years, with women representing $86.2 \%$ of these patients, and $76.4 \%$ of all patients were Caucasian (vs. $14.5 \%$ Hispanics). Compared with white registrants, Hispanic registrants were significantly younger in age, had a higher proportion of females, were noted to have a higher mean model for end-stage liver disease score at listing, were more likely to develop complications related to portal hypertension at the time of listing, and were less likely to have private insurance. The authors found that the proportion of Hispanic registrants increased from $10.7 \%$ to $19.3 \%$ in this period of time, despite an overall decrease in the total number of PBC registrants; they also had the highest percentage of waitlist deaths, the lowest rate for undergoing LT, a significantly higher risk of death while on the waitlist, and the highest proportion of waitlist removals due to clinical deterioration. ${ }^{11}$

In the current study, Hispanics and non-Hispanics were not different with respect to the multiple risk factors and comorbidities thought to be associated with PBC and reported similar rates of pruritus and fatigue. Importantly, the main findings in the present study relate to differences in socioeconomic factors. Namely, Hispanics with PBC were less likely to be married $(58 \%$ vs. $75 \%)$, indicating less help with responsibilities at home. These patients also had a lower level of education and lower income, and as expected were less likely to have medical insurance. All of these factors point towards more difficult access to health care among Hispanics, which could indeed explain the difference in disease severity noted by our group and by Peters et al. ${ }^{1,2}$ Decreased access to health care can potentially lead to late presentation, more severe disease and lack of response to available therapies. In addition, the fact that the vast majority of Hispanics were diagnosed with PBC only when living in the USA also raises concern for missed diagnosis at their country of origin and longer disease duration prior to diagnosis. Finally, given that there was no difference in the mean age at diagnosis, a delayed diagnosis among Hispanics would imply initial presentation at age younger than 50, and it is well known that age is a determinant of $\mathrm{PBC}$ progression and response to UDCA. ${ }^{12}$

Our study was limited by a relatively low response rate, the potential for recall bias and the inability to provide strong evidence of cause and effect, as is characteristic of survey studies. To enhance our response rate, we attempted to contact patients through three different methods (mail, email, and phone). It is possible that a lower income was also associated with decreased access to communication, in the form of e-mail or phone; if that is the case, lower response rates might be expected in this subgroup of patients. For others, legal status in this country could also play a role in their willingness to participate in a research study, and this in turn could have affected the response rates of the immigrant population. Trained personnel conducted all of the phone interviews in a standardized fashion. In addition, when appropriate, these interviews were conducted by a native Spanish speaking investigator to improve patients' level of comfort with the study.

We also acknowledge that there was not sufficient information on the prevalence of NAFLD or NASH and its effect on progression of PBC in this cohort of patients.

It has been reported that both Hispanics and African Americans have a more severe presentation of PBC compared to Caucasian patients. In the analysis by Peters et al., ${ }^{1}$ when comparing with Caucasian patients, the African American and Hispanic patients were significantly more likely to be limited in their physical activity level, to have severe or difficult-tocontrol pruritus, or to have a history of ascites, hepatic encephalopathy, or variceal bleeding. ${ }^{1}$ Ethnic disparities are also evident in terms of in-hospital mortality for PBC patients; despite a reported increase in hospitalizations of non-Hispanic PBC patients from $57.8 \%$ to $71.2 \%$ in a 7 -year period (from 2007 to 2014 ) compared to $4.1-6.3 \%$ for AfricanAmericans and 8.6-10.9\% for Hispanics, the highest in-hospital mortality was observed in African-American PBC patients. ${ }^{14}$ In the present study, both Caucasian and African American non-Hispanics were analyzed together; although this could result in an overestimation of severity for the non-Hispanic group, it is unlikely to have a significant effect in our study, given that only $3.1 \%$ of the non-Hispanic patients self-identified as African American.

To our knowledge, this is the first study to compare risk factors and comorbidities between Hispanic and non-Hispanic patients with PBC. Our results should be validated in a larger cohort.

In summary, we have shown that access to health care was lower in the Hispanic population compared to the nonHispanic population with PBC, which at least in part explains the differences in disease severity seen in previous studies. Environmental risk factors and comorbidities do not seem to significantly explain the variability in the disease course in different ethnic populations. Future studies should focus on evaluating different genetic backgrounds between Hispanic 
and non-Hispanic patients with PBC and its role in the severity and progression of PBC.

\section{Funding}

None to declare.

\section{Conflict of interest}

The authors have no conflict of interests related to this publication.

\section{Author contributions}

Designed the study and wrote the manuscript (AR), (CL) performed the study and analyzed the data (AR, NAPP, AFDLV, CL).

\section{References}

[1] Peters MG, Di Bisceglie AM, Kowdley KV, Flye NL, Luketic VA, Munoz SJ, et al. Differences between Caucasian, African American, and Hispanic patients with primary biliary cirrhosis in the United States. Hepatology 2007;46:769-775. doi: 10.1002/hep.21759.

[2] Levy C, Naik J, Giordano C, Mandalia A, O'Brien C, Bhamidimarri KR, et al. Hispanics with primary biliary cirrhosis are more likely to have features of autoimmune hepatitis and reduced response to ursodeoxycholic acid than non-Hispanics. Clin Gastroenterol Hepatol 2014;12:1398-1405. doi: 10. 1016/j.cgh.2013.12.010.

[3] Aragon TJ, Fay MP, Wollschlaeger D, Omidpanah A. Epitools: Epidemiology tools. Available from: https://cran.r-project.org/web/packages/epitools/index.html.
[4] Carrion AF, Ghanta R, Carrasquillo $O$, Martin P. Chronic liver disease in the Hispanic population of the United States. Clin Gastroenterol Hepatol 2011;9: 834-841. doi: 10.1016/j.cgh.2011.04.027.

[5] Parikh-Patel A, Gold EB, Worman H, Krivy KE, Gershwin ME. Risk factors for primary biliary cirrhosis in a cohort of patients from the united states. Hepatology 2001;33:16-21. doi: 10.1053/jhep.2001.21165.

[6] Corpechot C, Chrétien Y, Chazouillères O, Poupon R. Demographic, lifestyle, medical and familial factors associated with primary biliary cirrhosis. Hepatol 2010;53:162-169. doi: 10.1016/j.jhep.2010.02.019.

[7] Gershwin ME, Selmi C, Worman HJ, Gold EB, Watnik M, Utts J, et al. Risk factors and comorbidities in primary biliary cirrhosis: a controlled interview-based study of 1032 patients. Hepatology 2005;42:1194-1202. doi: 10.1002/hep.20907.

[8] Prince MI, Ducker SJ, James OF. Case-control studies of risk factors for primary biliary cirrhosis in two United Kingdom populations. Gut 2010;59: 508-512. doi: 10.1136/gut.2009.184218.

[9] Lammert C, Juran BD, Schlicht E, Chan LL, Atkinson EJ, de Andrade M, et al. Biochemical response to ursodeoxycholic acid predicts survival in a North American cohort of primary biliary cirrhosis patients. J Gastroenterol 2014 49:1414-1420. doi: 10.1007/s00535-013-0903-1.

[10] Corpechot C, Gaouar F, Chrétien Y, Johanet C, Chazouillères O, Poupon R. Smoking as an independent risk factor of liver fibrosis in primary biliary cirrhosis. J Hepatol 2012;56:218-224. doi: 10.1016/j.jhep.2011.03.031.

[11] Cholankeril G, Gonzalez HC, Satapathy SK, Gonzalez SA, Hu M, Khan MA, et al. Increased waitlist mortality and lower rate for liver transplantation in hispanic patients with primary biliary cholangitis. Clin Gastroenterol Hepatol 2018;16:965-973.e2. doi: 10.1016/j.cgh.2017.12.017.

[12] Carbone M, Mells GF, Pells G, Dawwas MF, Newton JL, Heneghan MA, et al. Sex and age are determinants of the clinical phenotype of primary biliary cirrhosis and response to ursodeoxycholic acid. Gastroenterology 2013; 144:560-569.e7. doi: 10.1053/j.gastro.2012.12.005.

[13] Podda M, Selmi C, Lleo A, Moroni L, Invernizzi P. The limitations and hidden gems of the epidemiology of primary biliary cirrhosis. J Autoimmun 2013;46: 81-87. doi: 10.1016/j.jaut.2013.06.015.

[14] Galoosian A, Hanlon C, Tana M, Cheung R, Wong RJ. Race/ethnicity and insurance-specific disparities in in-hospital mortality among adults with primary biliary cholangitis: Analysis of 2007-2014 national inpatient sample. Dig Dis Sci 2020;65:406-415. doi: 10.1007/s10620-019-05809-x. 